\title{
Does Cervicogenic Headache Result in the Presence of Neural Tension, and Does this Affect the Position and Mobility of Atlas?
}

\author{
Rob Sillevis ${ }^{* 1}$, Eric Shamus ${ }^{2}$ and Karen Wyss ${ }^{3}$
}

\author{
${ }^{1}$ Assistant Professor, Florida Gulf Coast University, Fort Myers, \\ Florida, Unites States \\ ${ }^{2}$ Professor, Florida Gulf Coast University, Fort Myers, Florida, Unites \\ States
}

${ }^{3}$ Physical therapist private practice, Integrated Therapy Practice PC, Hobart, Indiana, Unites States

\author{
${ }^{*}$ Corresponding author \\ Rob Sillevis, Assistant Professor, Florida Gulf Coast University, Fort Myers, \\ Florida, Unites States
}

Submitted: 09 Dec 2020; Accepted: 15 Dec 2020; Published: 05 Jan 2021

\begin{abstract}
Background: Cervicogenic headaches (CGH) can be the result of dysfunction of the upper cervical spine. Due to the soft tissue connection between muscle, cervical fascia, and dura, this region might contribute to the development of CGH.

Objective: Evaluate if subjects with CGH have neural tension signs. The secondary objective was to investigate if a correlation between the position and mobility of atlas and cervicogenic headaches exists.

Methods: 60 Subjects were recruited. Self-reported outcome measures, passive neck flexion rotation test, upper limb tension test (ULTT), slump test, and straight leg raise test (SLR) were assessed.

Results: There was a significant difference in atlanto-axial rotation to the right with $p=0.025$. There was no statistical significance in left rotation. There was no significant relationship between CGH, ULTT, slump and SLR with P>0.05. Atlas position was significantly related to CGH with $P<0.001$, and position of the atlas was significantly related to atlanto-axial motion with $p<0.001$. Discussion: There is a direct relationship between the position and mobility of atlas and CGH. Fascial connections between structures could result in dural tension and should be considered when managing patients with CGH. The use of the ULTT, Slump test, and SLR test does not appear to be beneficial in identifying those with CGH.
\end{abstract}

Keywords: Cervicogenic Headache, Atlas Positional Fault, Slump Test, Upper Limb Tension Test, Straight Leg Raise, Neural Tension.

\section{Introduction}

Neck disorders are common, and the healthcare system's burden is more than 50 billion dollars each year [1,2]. Individuals with dysfunctions originating in the cervical spine can present with a large variety of symptomatic regions, including the neck, head and face, shoulder, arm, thoracic spine, and scapular region. Cervicogenic headaches (CGH) are caused by dysfunction in the upper cervical spine. CGH typically present as a unilateral headache and are considered a secondary headache. A source in the neck will cause pain in 1 or more regions in the head and or face [3]. The prevalence of CGH has been reported to range from $15 \%$ to $20 \%$ of all headaches [4]. Around $4 \%$ of the general population experiences CGH $[5,6]$. Although the exact mechanism remains elusive, it appears that $\mathrm{CGH}$ are the result of mechanical dysfunction in the upper cervical spine [6-8]. The direct relationship between the trigeminal nerve and the spinal nerves $\mathrm{C} 1$ through $\mathrm{C} 3$ at the trigeminocervical nucleus might explain the development of CGH $[9,10]$. The trigeminocervical nucleus is functionally continuous with the dorsal horns of the upper cervical segments; therefore, nociceptive signals can converge with the trigeminal secondorder neuron. Referred pain through the ophthalmic branch of the trigeminal nerve might explain the subjective perception of orbital [9-11].

Another rationale for the development of CGH might be related to the fact that the motion of the suboccipital region is controlled by several groups of both smaller and larger muscle groups. Abnormal muscle tone can lead to abnormal cervical motion patterns and alter the proprioceptive information received from the mechanoreceptors in this region [12]. There is an exceptionally high density of mechanoreceptors in the suboccipital muscles in the posterior upper cervical region [12]. Cervical dysfunction can 
result in pain, capsule irritation, and abnormal muscle tone and functioning. Fascial tissue connections between the rectus capitis posterior minor, rectus capitis posterior major, and the obliquus capitis inferior and the dura have been identified previously [13, 14]. These fascial tissue connections have been referred to as the "myodural bridges". The myodural bridges merge with the meningiovertebral ligaments between the arches of $\mathrm{C} 1$ and $\mathrm{C} 2$, cross the epidural space, and insert into the posterior aspect of the dura mater $[13,14]$. It has been proposed that these myodural bridges protect the cervical dura from compression during motion [13]. The myodural bridge's significance is that there is a direct anatomical connection between the muscle, cervical fascia, and the central nervous system. This connection between the suboccipital musculature and the dura could lead to neural tension. Neural tension has been identified by clinicians when treating patients with headaches and cervical related dysfunctions [15-17].

Muscular impairment (dysfunctions) can be a possible contributing factor for the development of $\mathrm{CGH}[18,19]$. The upright position in humans results in a vertical gravitational compressive force in the cervical spine due to the weight of the head. Over time this could result in progressive degenerative changes in the cervical spine. These changes will result in added mechanical compression due to an increased cervical curve and a posterior rotation of the head. The ventral ramus of the upper cervical spine innervates the prevertebral muscles along with the facet capsules of C2 and C3 [20]. Additionally, the trapezius and sternocleidomastoid (SCM) receive innervation through the upper cervical spine. The joint capsules of the atlantoaxial joints, the stabilizing ligaments, and the dura mater are also supplied by $\mathrm{C} 1-\mathrm{C} 3$ nerves $[20,21]$. Based on function and position, muscles will adapt. Patients with $\mathrm{CGH}$ typically present with forward head posture leading to muscle adaptation. This muscle adaptation pattern has been described as the upper cross muscle syndrome (UCMS) [22]. It is characterized by the concurrent development of weak and short muscles. In subjects with $\mathrm{CGH}$, muscle weakness has been identified in the deep neck flexor muscles. Muscle imbalance might increase the susceptibility to injury in the cervical region [23]. Within this UCMS, the sternocleidomastoid, upper trapezius, scalene, and suboccipital muscles will present in a hypertonic state and shortened position [18]. The shortening and increased tone of the suboccipital muscles could result in prolonged neural tension through the myodural bridge.

It has been demonstrated that $\mathrm{CGH}$ are caused by dysfunction of the upper cervical spine. Due to the soft tissue connection between muscle, cervical fascia, and dura this region might contribute to the development of CGH. Therefore, this study's primary aim was to evaluate if subjects with $\mathrm{CGH}$ have concurrent neural tension signs. The secondary aim of this study was to investigate if there is a correlation between the position of atlas and mobility of the atlantoaxial joint in those experiencing cervicogenic headaches compared to a healthy control group.

\section{Methods}

Subjects

A convenience sampling method was used to recruit our subjects from a physical therapy clinic in Northwest Indiana. Based on a power analysis using a power of $80 \%$, alpha of 0.05 , and an effect size of 0.4 , it was determined to recruit 60 subjects for this study. All available subjects were screened for eligibility criteria. All subjects had to be between the ages of 18 and 65, be able to speak and read the English language fluently, and have either cervicogenic headaches or no experiences of headaches. The CGH group subjects had to have a neck disability index (NDI) greater than 15 . To qualify for the non-headache control group, the subjects were not to have any cervical related diagnosis or neck pain, or experience any headaches at the time of testing. The subjects were screened for any red flags and potential reasons for not undergoing the testing protocol by the treating physical therapist. Additional exclusion criteria constituted evidence of central nervous system involvement, including hyperreflexia, nystagmus, loss of visual acuity, an impaired sensation of the face, altered taste, and the presence of pathological reflexes. This study received institutional review board (IRB) approval from Florida Gulf Coast University.

\section{Testing Protocol}

All 60 subjects (30 subjects in the CGH group and 30 subjects in the non-headache control group) were tested after giving written consent as part of their initial physical therapy evaluation. Each subject completed the self-reported outcome measures first (Visual Analogue Scale, Neck Disability Index, and Headache Disability Invetory), after which the passive neck-flexion-rotation test (FRT) and passive neural tension tests were randomly administered at the discretion of the physical therapist. The evaluating physical therapists were blinded to the results of the self-reported outcome measures.

\section{Self-Reported Outcome Measures}

Before the physical examination, each subject completed the visual analog scale (VAS) to assess neck pain at the time of the evaluation [24]. Subjects marked their pain at the time of the initial evaluation on a 10-centimeter scale. The left side of the scale is identified as "no pain at all" and the right side as "worst pain imaginable." The distance in millimeters from zero is recorded. The validity and reliability of the VAS for patients with acute and chronic pain have been reported as being good [25]. To get an impression about the self-perceived functioning of the cervical spine and the headaches, both the Neck Disability Index (NDI) and the Headache Disability Inventory (HDI) were used. The NDI is a 10 -item patient selfreported measure used to identify each subject's level of reported disability [26-30]. Higher scores on the NDI indicate greater disability levels [31]. The NDI has good validity and reliability in patients with neck pain related conditions $[26,32]$. The HDI is a 25-item patient self-reported measure evaluating the direct impact of headaches on daily living. Higher scores indicate higher impact of the headaches. There are two scales, including 12 functional and 13 emotional items, combined for a maximum total score of 100. The construct validity and the test-retest reliability of the HDI have been demonstrated as good [33]. The HDI is highly reliable with values ranging between 0.93 and 0.95 [33]. The NDI has also been shown to have good content and construct validity and excellent test-retest reliability of 0.96 [33].

\section{Atlantoaxial Range of Motion Assessment and Atlas Position}

All 60 subjects underwent an assessment of the mobility of the upper cervical region. The FRT was used to determine rotation at the atlanto-axial (AA) joint. During the FRT, the cervical spine is flexed maximally. In that position, rotation of the head in both 
directions was measured with the goniometer and assessed for the quality of end-feel [34]. The FRT is reliable in patients with cervicogenic headaches [35]. Based on high sensitivity (90 to $91 \%$ ), specificity (88 to $90 \%$ ), and overall diagnostic accuracy $(91 \%)$, the FRT is an appropriate test to use clinically $[35,36]$.

The position of atlas relative to the occiput was determined using direct palpation of atlas' transverse process. The subjects were graded as neutral or "normal" if there was no observable difference in the position of bilateral transverse processes (TP) of the atlas. The TPs were palpated while the subjects were seated in a comfortable, natural position while maintaining a mandible protrusion. The presence of a right rotation default position of the atlas was assumed if there was a relative anterior position of the left transverse process compared to the right. A left rotation positional default position of the atlas was assumed if there was a relative anterior position of the right transverse process compared to the left transverse process. The positional default position of the atlas has been proposed previously and demonstrated to exist by using musculoskeletal ultrasound [37].

\section{Assessment of Neural Tension}

Neural tension was assessed using the slump test, upper limb tension test A (ULTT), and the straight leg raise test (SLR). All three tests are commonly used in the management of patients with musculoskeletal dysfunctions. The slump test was performed with the subjects seated with the entire spine in a slumped position and the neck in maximal forward flexion. The subject's hands were positioned behind the pelvis. Starting with one knee at 90 degrees of flexion with maximal dorsal flexion of the ankle, the knee was actively extended to the point of discomfort. At this point, the subject actively extended the neck. The test was considered positive if the symptoms in the leg disappear. As a measurement of a positive slump test, the knee flexion angle provoking symptoms was measured. The slump test was then repeated on the other side. Intra-rater reliability has been reported as good with an ICC of
0.95 [38]. Sensitivity and specificity of the slump test has been reported as being 0.84 and 0.83 respectively [39].

The ULTT is a neural tension test carried out in the supine position. The subject's scapula was held in a somewhat depressed position by the physical therapist; at the same time, the following movements of the arm were passively added until symptom reproduction occurred (shoulder abduction followed by forearm supination, wrist extension, finger extension, shoulder lateral rotation, and elbow extension). After this, passive cervical side bending to the contralateral side was added until symptoms occur. When there were symptoms, ipsilateral passive cervical side bending was added to decrease symptoms. The angle of elbow extension provoking pain and or symptoms served as the measure of a positive ULTT. The ULTT test has been identified as an excellent test to rule in neural tension with a specificity of 0.97 and an inter-rater reliability kappa value of 0.76 [40].

The SLR test was carried out in the supine position. The subject fully extended the knee with the ankle in a neutral position. The physical therapist then passively flexed the hip while maintaining knee extension. Reproducing symptoms of pain and or tightness subsiding on plantar flexion of the ankle was considered a positive test. The angle of hip flexion was measured with a goniometer at the point of symptoms. The sensitivity of the SLR is reported at 0.87 and the specificity at $0.89[39,41]$.

\section{Statistical Analysis}

Statistical analyses were performed using the SPSS, version 26.0, statistical software package. The data were analyzed for normal distribution using the Sharpo-Wilk test. The HDI and the neck flexion rotation test were normally distributed, so the independent t-test was used to determine the difference between the headache and the non-headache control group. The NDI, Slump, ULTT, and the SLR were not normally distributed. Therefore, non-parametric statistics were used to analyze the data (Table 1).

Table 1. Test of Normality.

\begin{tabular}{|l|c|c|c|c|c|c|}
\hline \multicolumn{7}{|c|}{ Tests of Normality } \\
\hline & \multicolumn{2}{|c|}{ Kolmogorov-Smirnova } & \multicolumn{3}{c|}{ Shapiro-Wilk } \\
\hline & Statistic & $\mathrm{df}$ & Sig. & Statistic & df & Sig. \\
\hline NDI & 137 & 31 & 143 & .923 & 31 & 028 \\
\hline HDI & 113 & 31 & $200^{*}$ & .978 & 31 & 755 \\
\hline VAS & 087 & 31 & $200^{*}$ & .975 & 31 & 660 \\
\hline SlumpR & 473 & 31 & 000 & .495 & 31 & 000 \\
\hline SlumpL & 512 & 31 & 000 & .415 & 31 & 000 \\
\hline ULTTR & 458 & 31 & 000 & .351 & 31 & 000 \\
\hline ULTTL & 493 & 31 & 000 & .358 & 31 & 000 \\
\hline SLRR & 376 & 31 & 000 & .601 & 31 & 000 \\
\hline SLRL & 388 & 31 & 000 & .622 & 31 & .000 \\
\hline NFRTR & 124 & 31 & $200^{*}$ & .978 & 31 & 757 \\
\hline NFRTL & 109 & 31 & $200^{*}$ & .955 & 31 & 217 \\
\hline
\end{tabular}

*. This is a lower bound of the true significance.

a. Lilliefors Significance Correction 


\section{Baseline Characteristics}

A total of 60 subjects were assessed for eligibility and enrolled in the study. Twenty-six subjects were male $(43.3 \%)$, while 34 were female $(56.7 \%)$, and the mean subject age was 48.5 , with a range of 20 to 65 years (Table 2).

Table 2. Descriptive statistics study sample.

\begin{tabular}{|l|c|c|c|c|c|}
\hline \multicolumn{7}{|c|}{ Descriptive Statistics } \\
\hline & $\mathbf{N}$ & Minimum & Maximum & Mean & Std. Deviation \\
\hline Gender & 60 & 1.00 & 2.00 & 1.5667 & .49972 \\
\hline age & 60 & 20.00 & 65.00 & 48.4833 & 13.28245 \\
\hline condition & 60 & 1.00 & 2.00 & 1.5000 & .50422 \\
\hline HDI & 31 & .00 & 74.00 & 36.6452 & 17.74739 \\
\hline NDI & 31 & 14.00 & 56.00 & 29.7097 & 11.98664 \\
\hline VAS & 53 & 00 & 99.00 & 36.7170 & 29.58584 \\
\hline SlumpR & 60 & 00 & 27.00 & 2.6167 & 6.64370 \\
\hline SlumpL & 60 & 00 & 22.00 & 1.6333 & 4.71193 \\
\hline ULTTR & 60 & 00 & 90.00 & 3.1167 & 12.92494 \\
\hline ULTTL & 60 & 00 & 55.00 & 2.5667 & 8.42186 \\
\hline SLRR & 60 & 35.00 & 90.00 & 81.7167 & 14.78787 \\
\hline SLRL & 60 & 40.00 & 90.00 & 81.7000 & 13.75118 \\
\hline Atlas & 60 & 1.00 & 3.00 & 1.8333 & .76284 \\
\hline NFRTR & 60 & 15.00 & 70.00 & 34.6667 & 10.09223 \\
\hline NFRTL & 60 & 15.00 & 60.00 & 36.0000 & 9.82042 \\
\hline
\end{tabular}

HDI=Headache Disability Inventory, NDI=Neck Disability Index, VAS= Visual Analogue Scale, SlumpR $=$ slump test on the right, SlumpL= slump test on the left, ULTTR= upper limb tension test right, ULTTL= upper limb tension test left, SLRR= straight leg raise test right, $\mathrm{SLRL}=$ straight leg raise test right, $\mathrm{NFRTR}=$ neck flexion rotation test right, $\mathrm{NFRTL}=$ neck flexion rotation test left.

\section{CGH and Neck Flexion Rotation Test}

To determine the difference in the neck flexion rotation test between the CGH group and the non-headache control group, the cervical rotation range of motion in both groups was compared for each side separately. The independent t-test was utilized. There was a significant difference in the amount of passive AA rotation to the right between the $\mathrm{CGH}(\mathrm{M}=31.77, \mathrm{SD}=6.7, \mathrm{SEM}=$ $1.28)$ and the control group $(\mathrm{M}=31.57, \mathrm{SD}=11.87, \mathrm{SEM}=2.17)$; $\mathrm{t}(58)=-2.306, \mathbf{p}=\mathbf{0 . 0 2 5}$. There was a difference in the amount of passive AA rotation to the left between the $\mathrm{CGH}(\mathrm{M}=33.77$, SD $=9.53, \mathrm{SEM}=1.74)$ and the control group $(\mathrm{M}=38.23, \mathrm{SD}=9.75$, $\mathrm{SEM}=1.78)$; however, this was not statistically significant; $\mathrm{t}(58)$ $=-1.794, \mathbf{p}=\mathbf{0 . 0 7 8}$.

\section{CGH and upper limb tension test}

To determine the difference in the upper limb tension between the CGH group and the non-headache control group, the angle of elbow flexion in both groups was compared for each side separately. The Mann Whitney U test was utilized. There was no significant difference between the ULTT elbow flexion measurement on the right between the CGH and the control group: $\mathrm{U}=413.5, \mathbf{p}=\mathbf{0 . 3 6 1}$. There was no significant difference between the ULTT elbow flexion measurement on the left between the $\mathrm{CGH}$ and the control group: $\mathrm{U}=424, \mathbf{p}=\mathbf{0 . 5 1 5}$.

\section{CGH and SLR test}

To determine the difference in the SLR test between the CGH group and the non-headache control group, the angle of hip flexion in both groups was compared for each side separately. The Mann Whitney $\mathrm{U}$ test was utilized. There was no significant difference between the passive SLR hip flexion measurement on the right between the $\mathrm{CGH}$ and the control group: $\mathrm{U}=425, \mathbf{p}=\mathbf{0 . 6 6 4}$. There was no significant difference between the passive SLR hip flexion measurement on the left between the $\mathrm{CGH}$ and the control group: $\mathrm{U}=422, \mathbf{p}=\mathbf{0 . 6 2 7}$.

\section{CGH and Slump test}

To determine the difference in the Slump test between the CGH headache group and the non-headache control group, the angle of knee flexion in both groups was compared for each side separately. The Mann Whitney U test was utilized. There was no significant difference between the Slump knee flexion angle on the right between the CGH and the control group: $U=420, \mathbf{p}=\mathbf{0 . 4 9 4}$. There was no significant difference between the Slump knee flexion angle on the left between the $\mathrm{CGH}$ and the control group: $\mathrm{U}=447$, $\mathbf{p}=\mathbf{0 . 9 4 0}$.

\section{Correlation Between Outcome Measures and Neural} Tension Testing

To determine the correlation between the FRT, ULTT, SLR, and 
the Slump test, the Pearson Correlation was utilized. There was a small correlation between the Slump test and the ULTT (right $\mathrm{r}=0.15$, left $\mathrm{r}=0.23$ ). There was a medium correlation between the Slump test on the right and the SLR on the right; $r=-0.44$. There was a large correlation between the Slump test on the left and the SLR left; $\mathrm{r}=-0.51$. There was a small correlation between the FRT and the ULTT (right $\mathrm{r}=0.1$, left $\mathrm{r}=0.23$ ). There was a small correlation with the Slump test on the right and no correlation on the left side (right $\mathrm{r}=-0.1$, left $\mathrm{r}=0.03$ ). There was a small correlation with the SLR test (right $r=-0.21$ and left $r=-0.31$ ). The HDI results were not related to the FRT (right $\mathrm{r}=0.04$, left $\mathrm{r}=0.08$ ). There was a small correlation with the Slump test on the right $(\mathrm{r}=-0.252)$ and no correlation on the left $(\mathrm{r}=0.02)$. There was a small correlation with the ULTT (right $\mathrm{r}=-0.22$, left $\mathrm{r}=-0.308$ ). There was a small correlation with the SLR (right $r=-0.291$, left $r=-0.184$ ). There was a small correlation between the NDI and the HDI $(\mathrm{r}=0.196)$.

\section{Correlation between Atlas position and CGH, and FRT}

To determine if the position of atlas was correlated with the presence of CGH, the chi-square test of independence was performed. The relationship between the atlas position and CGH was significant, $\mathrm{X} 2=20.526, \mathrm{P}<0.001$. Subjects with a rotated atlas upon palpation were more likely to experience CGH.

To determine the relationship between the atlas position and the mobility of the AA joints test through the FRT, the Kruskal-Wallis test was performed. It appears that the atlas position significantly affects the AA mobility measured in the FRT, H (2) $=18.55$, p - $\cap \cap 1 \quad(\Gamma: \ldots . . .1$
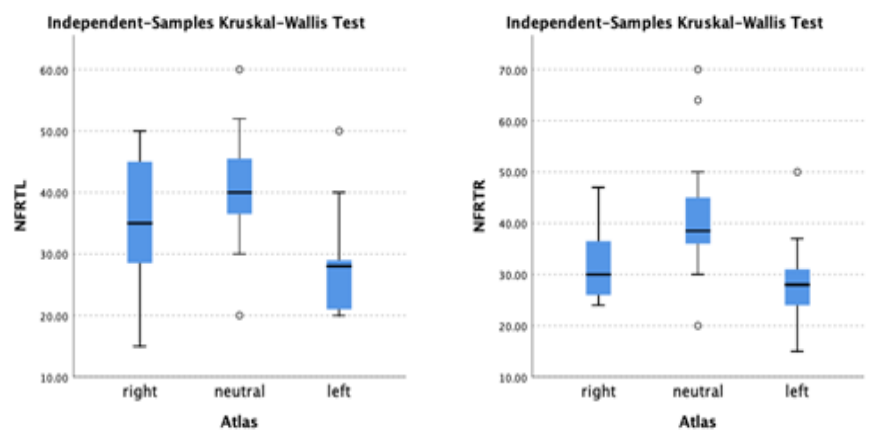

Table 3: Kruskal-Wallis results: atlas position related to AA mobility

\section{Discussion}

The primary aim of this study was to evaluate if subjects with CGH have concurrent neural tension signs. The secondary aim was to investigate if there is a correlation between the position of atlas and mobility of the atlantoaxial joint in those experiencing cervicogenic headaches compared to a healthy control group. Cervicogenic headache is defined as a headache that is the results from cervical related dysfunction and perceived in 1 or more regions in the head and or face. 3 The current rationale explaining the mechanism of how CGH develop is through the neurogenic relationship between the spinal nerves $\mathrm{C} 1$ through $\mathrm{C} 3$ and the trigeminal nerve at the trigeminocervical nucleus.9,10 This is where the nociceptive signals of the upper cervical joints will converge with the trigeminal second-order neuron and result in sensitization of the trigeminal nerve. Cervical spinal nerves below $\mathrm{C} 3$ have not shown to radiate toward any region of the head [42]. This study support that the spinal segments below $\mathrm{C} 4$ do not contribute to the development of CGHs. The ULTT places the C5-T1 cervical nerve roots under direct tension. This study demonstrates a weak and non-significant relationship between CGHs and the ULTT.

Meningiovertebral connections have been identified throughout the spinal column [43]. It has been hypothesized that the function of the meningiovertebral bridge is to maintain the spinal cord's position within the spinal canal during motion in such a way that there will be no pinching or compression of the cord during movement of the neck and head. The motion of the suboccipital region is controlled by several groups of both smaller and larger muscles. The suboccipital muscles are part of the complex control mechanism allowing motion of the atlas relative to the axis. During neck rotation, the head moves relatively on the neck, allowing the atlas to turn on the axis through the unilateral contraction of the rectus capitis major and the obliques capitis inferior. Myodural bridges in the upper cervical spine between the rectus capitis major and the obliquus capitis inferior muscles and the dura have been identified; however, the actual clinical relevance remains elusive $[43,44]$. When the head is turned, the myodural bridge will pull the dura mater ipsilaterally. Movement of the dura could lead to increased neural tensioning. This tension might play a role in the development of CGHs. This study's results support this possible secondary pathway of developing CGH as the FRT and the position of atlas were directly related to the $\mathrm{CGH}$.

Abnormal mechanical functioning of the upper cervical spine will change the proprioceptive awareness coming from this region [12]. Proprioception is the information sent by mechanoreceptive neurons to the brain, informing it of the position of the neck, head, and any positional changes. There is a high density of the mechanoreceptors in the suboccipital muscles in the posterior upper cervical spine [12]. Abnormal proprioception will lead to abnormal muscle tone. If suboccipital muscle contraction or tonicity is maintained for a prolonged period, this could result in a change in positional relationships between segments of the upper cervical spine. Such positional default in a subject with CGH has been previously suggested and confirmed with musculoskeletal ultrasound imaging by Sillevis and Swanick.37 In this study, palpation was used to identify the relative position of atlas, and the results of this study support the hypothesis that a positional default of atlas in subjects with CGH was present. If a positional default position is a contributing factor in the development of $\mathrm{CGH}$, it cannot be determined based on this study. However, it can be hypothesized that an increase in muscle tightness would result in decreased mobility. This study supports this notion as the CGH group had a significantly decreased right rotation of the atlantoaxial joint compared to the control group. There was a difference but not significant with left rotation. The inter and intra-rater reliability of palpation for the position of the atlas has not been reported previously, and this could have been a limiting factor in this study.

Scali et al and Pontel et al previously suggested that increased tension of the suboccipital muscles could lead to $\mathrm{C} 2$ nerve tension due the myodural bridge's presence $[13,14]$. Due to these fascialike connections, muscle tone could affect the functioning of the 
dura and possibly negatively affect dural mobility. Neural tensions tests are clinically used to identify if the movement of neural tissues is normal by progressively increasing the pull on these tissues. If there are limitations in neural mobility, direct pull will provoke patient-related symptomology. In this study, we used the bilateral ULTT, Slump test, and the bilateral SLR tests to aasess if neural mobility was restricted in subjects with CGH. It appears that the results of this study cannot support the do not support the notion that there is restricted mobility in the upper cervical dura. This study demonstrated that there was a weak correlation between the ULTT and the presence of CGH. This weak correlation could be explained by the fact that the nerves that make up the brachial plexus leave the spinal cord below the C4 level. The Slump test and the SLR test were used to load the nervous system in the sagittal plane from head to toe. There was no significant relationship identified between CGH and either the Slump and or the SLR test. What was not surprising was the medium to large correlation between the slump tests and the SLR test supporting the thought that both tests have a similar effect of the dura. The fact that both the Slump and the SLR do not correlate with CGH might be due to the fact that the meningiovertebral bridges in the lumbar and thoracic spine might prevent direct pull on the upper cervical dural during movement.43 The hypothesis that the CGH are the result of pathophysiology in the upper cervical spine is supported by the fact that our study results only demonstrate small correlations between the ULTT, the Slump test, the SLR test, and CGH's. This study has several limitations. It is possible that the nonheadache control group had musculoskeletal conditions that could have directly affected the mobility of the nervous system and, thus, affected the neural tension tests. Another limitation that could have affected the outcome of this study is that we did not identify if the subject was right or left-handed. Handedness might coincide with the preference of neuromuscular pathways in the high cervical spine and, thus, affect AA mobility and, therefore, affect dural tension. Our subjects were not assessed for forward head posture. This position could lead to a posterior rotation of the occiput on the atlas in the sagittal plane. Forward head posture will place the suboccipital muscles and the posterior neck muscles in a shortened position resulting in muscle hypertrophy. The presence of the myodural bridge might then result in direct neural tension and could have affected the NFRT mobility testing [43, 45].

\section{Conclusion}

This study supports the hypothesis that there is a direct relationship between the position of atlas, unilateral restriction in AA rotation, and CGH presence. The presence of fascial connections between structures of the high cervical spine and the dura motion could result in dural tension and should be considered by physical therapists when managing patients with CGH. The use of the ULTT, Slump test, and SLR test does not appear beneficial in identifying those with CGH. Further research is necessary to evaluate the relationship and clinical relevance of the myodural bridge in the upper cervical spine and the possible causative relationship with the development of $\mathrm{CGH}$.

\section{References}

1. Strine T, Hootman J (2007) US National Prevelance and Correlations of Low Back and Neck Pain Among Adults. Arthritis \& Rheumatism (Arhtritis Care \& Research) 57: 656665.
2. Taylor H, Murphy B (2008) Altered Sensorimotor Integration with Cervical Spine Manipulation. Journal of Manipulative and Physiological Therapeutics 31: 116-125.

3. Headache Classification Subcommittee of the International Headache Society (2004) The International Classification of Headache Disorders: 2nd edition. Cephalalgia 24: 9-160.

4. Nilsson N (1995) The prevalence of cervicogenic headache in a random population sample of 20-59 year olds. Spine 20: 1884-1888.

5. Sjaastad O, Bakketeig L (2008) Tension-type headache: Comparison with migraine without aura and cervicogenic headache. The Vaga study of headache epidimiology. Functional Neurology 23: 71-76.

6. Sjaastad O, Bakketeig L (2008) Prevelance of cervicogenic headache: VAGA study of hedache epidemiology. Acta Neurol Scand 117: 173-180.

7. Haldeman S, Dagenais S (2001) Cervicogenic headaches: a critical review. The Spine Journal 1:31-46.

8. Aprill C, Axinn M, Bogduk N (2002) Occipital headcahes stemming from the lateral atlanto-axial (C1-2) joint. Cephalalgia 22: 15-22.

9. Hall T, Briffa K, Hopper D (2008) Clinical evaluation of cervicogenic headache: a clinical perspective. J Man Manip Ther 16: 73-80.

10. Biondi DM (2001) Cervicogenic headache: diagnostic evaluation and treatment strategies. Curr Pain Headache Rep 5: 361-368.

11. Bogduk N (2014) The neck and headaches. Neurol Clin 32: 471-487.

12. Roijezon U, Clark NC, Treleaven J (2015) Proprioception in musculoskeletal rehabilitation. Part 1: Basic science and principles of assessment and clinical interventions. Man Ther 20: 368-377.

13. Scali F, Marsili ES, Pontell ME (2011) Anatomical connection between the rectus capitis posterior major and the dura mater. Spine (Phila Pa 1976) 36: E1612-1614.

14. Pontell ME, Scali F, Marshall E, Enix D (2013) The obliquus capitis inferior myodural bridge. Clin Anat 26: 450-454.

15. Szikszay TM, Luedtke K, Harry von P (2018) Increased mechanosensivity of the greater occipital nerve in subjects with side-dominant head and neck pain - a diagnostic casecontrol study. J Man Manip Ther 26: 237-248.

16. Caamano-Barrios LH, Galan-Del-Rio F, Fernandez-deLas-Penas C, Cleland JA, Plaza-Manzano G, et al. (2019) Evaluation of neurodynamic responses in women with frequent episodic tension type headache. Musculoskelet Sci Pract 44:102063.

17. von Piekartz HJ, Schouten S, Aufdemkampe G (2007) Neurodynamic responses in children with migraine or cervicogenic headache versus a control group. A comparative study. Man Ther 12: 153-160.

18. Moore MK (2004) Upper crossed syndrome and its relationship to cervicogenic headache. J Manipulative Physiol Ther 27: 414-420.

19. Biondi DM (2005) Noninvasive treatments for headache. Expert Rev Neurother 5: 355-362.

20. Bogduk N, Govind J (2009) Cervicogenic headache: an assessment of the evidence on clinical diagnosis, invasive tests, and treatment. Lancet Neurol 8: 959-968.

21. Bogduk N (1994) Innervation and Pain patterns of the Cervical 
Spine. In: Clinics in Physical Therapy: Physical Therapy of the Cervical and Thoracic Spine New York: Churchill Livingstone 1994: 65-76.

22. Fernandez-de-Las-Penas C, Ge HY, Arendt-Nielsen L, Cuadrado ML, Pareja JA (2007) Referred pain from trapezius muscle trigger points shares similar characteristics with chronic tension type headache. Eur J Pain 11: 475-482.

23. Petersen SM (2003) Articular and muscular impairments in cervicogenic headache: a case report. J Orthop Sports Phys Ther 33: 21-30.

24. Bird S, Dickson E (2001) Clinically Significant Changes in Pain Along the Visual Analoge Scale. Annals of Emergency Medicine 38: 639-643.

25. Gallagher E, Liebman M, Bijur P (2001) Prosepctive Validation of Clinically Important Changes in Pain Severity Measured on a Visual Analoge Scale. Annals of Emergency Medicine 38: 633-638.

26. Vernon H, Mior S (1991) The Neck Disability Index: a study of reliability and validity. Journal of Manipulative and Physiological Therapeutics 14: 409-415.

27. Childs J, Cleland J, Elliot J (2008) Neck Pain: Clinical Practice Guidelines Linked to the International Classification of Functioning, Disability, and Health from the Orthopaedic Section of the American Physical Therapy Association. Journal of Orthopaedic \& Sports Physical Therapy 38: A1A34.

28. Cleland JA, Childs JD, McRae M, Palmer JA, Stowell T (2005) Immediate effects of thoracic manipulation in patients with neck pain: a randomized clinical trial. Man Ther 10: 127135.

29. Tseng Y, Wang W, Chen W, Hou T, Chen T, et al. (2006) Predictors for immediate responders to cervical manipulation in patients with neck pain. Manual Therapy 11: 306-315.

30. Riddle D, Stratford P (1998) Use of generic versus regionspecific functional status measures on patients with cervial spine disorders. Physical Therapy 78: 951-963.

31. Cleland J, Fritz J, Whitman J, Palmer J (2006) The Reliability and Construct Validity of the Neck Disability Index and Patient Specific Functional Scale in Patients with Cervical Radiculopathy. Spine 31: 598-602.

32. Hoving J, O'Leary E, Niere K, Green S, Buchbinder R (2003) Validity of the neck disability index, Northwick Park neck pain questionnaire, and problem elicitation technique for measuring disability associated with whiplas-associated disorders. Pain 102: 273-281.
33. Jacobson GP, Ramadan NM, Norris L, Newman CW (1995) Headache disability inventory (HDI): short-term test-retest reliability and spouse perceptions. Headache 35 : 534-539.

34. Piva SR, Erhard RE, Childs JD, Browder DA (2006) Inter-tester reliability of passive intervertebral and active movements of the cervical spine. Man Ther 11: 321-330.

35. Hall TM, Robinson KW, Fujinawa O, Akasaka K, Pyne EA (2008) Intertester reliability and diagnostic validity of the cervical flexion-rotation test. J Manipulative Physiol Ther 31: 293-300.

36. Ogince M, Hall T, Robinson K, Blackmore AM (2007) The diagnostic validity of the cervical flexion-rotation test in C1/2-related cervicogenic headache. Man Ther 12: 256-262.

37. Sillevis R, Swanick K (2019) Musculoskeletal ultrasound imaging and clinical reasoning in the management of a patient with cervicogenic headache: a case report. Physiother Theory Pract 2019: 1-11.

38. Tucker N, Reid D, McNair P (2007) Reliability and measurement error of active knee extension range of motion in a modified slump test position: a pilot study. J Man Manip Ther 15: E85-91.

39. Majlesi J, Togay H, Unalan H, Toprak S (2008) The sensitivity and specificity of the Slump and the Straight Leg Raising tests in patients with lumbar disc herniation. J Clin Rheumatol 14: 87-91.

40. Wainner RS, Fritz JM, Irrgang JJ, Boninger ML, Delitto A, et al. (2003) Reliability and diagnostic accuracy of the clinical examination and patient self-report measures for cervical radiculopathy. Spine (Phila $\mathrm{Pa} 1976$ ) 28: 52-62.

41. Jonsson B, Stromqvist B (1995) The straight leg raising test and the severity of symptoms in lumbar disc herniation. A preoperative evaluation. Spine (Phila Pa 1976) 20: 27-30.

42. Becker WJ (2010) Cervicogenic headache: evidence that the neck is a pain generator. Headache 50: 699-705.

43. Scali F, Pontell ME, Nash LG, Enix DE (2015) Investigation of meningomyovertebral structures within the upper cervical epidural space: a sheet plastination study with clinical implications. Spine J 15: 2417-2424.

44. Shi B, Zheng X, Min S, Zhou Z, Ding Z, et al. (2014) The morphology and clinical significance of the dorsal meningovertebra ligaments in the cervical epidural space. Spine J 14: 2733-2739.

45. Enix DE, Scali F, Pontell ME (2014) The cervical myodural bridge, a review of literature and clinical implications. J Can Chiropr Assoc 58: 184-192.

Citation: Rob Sillevis, Eric Shamus and Karen Wyss (2021). Does Cervicogenic Headache Result in the Presence of Neural Tension, and Does this Affect the Position and Mobility of Atlas?. Journal of Medical \& Clinical Research 6(1):344-350. 